\title{
LncRNA IGFL2-ASI Promotes the Proliferation, Migration, and Invasion of Colon Cancer Cells and is Associated with Patient Prognosis
}

\author{
Xiaoning Cen ${ }^{1, *}$ \\ Yunmei Huang ${ }^{2, *}$ \\ Zhuangnian $\mathrm{Lu}^{3, *}$ \\ Wenjun Shao ${ }^{4}$ \\ Chenyi Zhuo (iD) ' \\ Chongchan Bao ${ }^{5}$ \\ Shi Feng ${ }^{5}$ \\ Cheng Wei ${ }^{5}$ \\ Xiqiang Tang ${ }^{5}$ \\ Lijun Cen (iD ${ }^{5}$ \\ Wenwen $\mathrm{Guo}^{5}$ \\ Xinru $\operatorname{Tian}^{5}$ \\ Qianli Tang (iD ${ }^{5}$ \\ Xusen Huang
}

'General Surgery, Affiliated Hospital of Youjiang Medical University for

Nationalities, Baise, 533000, Guangxi,

People's Republic of China; ${ }^{2}$ Department of Pathology, Affiliated Hospital of Youjiang Medical University for Nationalities, Baise, 533000, Guangxi, People's Republic of China; ${ }^{3}$ Department of Pediatrics, Affiliated Hospital of Youjiang Medical University for Nationalities, Baise, 533000, Guangxi, People's Republic of China; ${ }^{4}$ Medical college of Soochow University, Suzhou, Jiangsu, 215000, People's Republic of China; ${ }^{5}$ Youjiang Medical University for Nationalities, Baise, 533000, Guangxi,

People's Republic of China

*These authors contributed equally to this work

Correspondence: Qianli Tang Youliang Medical University for Nationalities, Baise, 533000, Guangxi,

People's Republic of China

Email htmgx@163.com

Xusen Huang

General Surgery, Affiliated Hospital of Youliang Medical University for

Nationalities, Baise, 533000, Guangxi,

People's Republic of China

Email hxsfy@I63.com
Background: LncRNAs play an important role in tumor initiation and development. However, the underlying involvement of lncRNA expression in colorectal carcinoma remains to be clarified.

Methods: All analyses were performed in R software v4.0, SPSS v13.0, and GraphPad Prism 8. The "limma" package was used to identify differentially expressed lncRNAs between two groups with the threshold of $|\log \mathrm{FC}|>1$ and $\mathrm{P}<0.05$. The "Survival" package was used to conduct survival analysis. HCT8 and SE480 cell lines were used to conduct further phenotype experiments, including transwell, wound-healing, CCK8 and colony formation assay. Gene set enrichment analysis was used to explore the biological pathway difference in high and low IGFL2-AS1 patients.

Results: The lncRNA IGFL2-AS1 was highly expressed in colon adenocarcinoma (COAD) tissue and cell lines (HCT116, HCT8, HCT129, and SW480). The COAD patients with high IGFL2-AS1 were associated with a worse prognosis. Meanwhile, the knockdown of IGFL2AS1 could significantly suppress the proliferation and invasion of COAD cells. Gene set enrichment analysis showed that the top five biological pathways involving IGFL2-AS1 were angiogenesis, epithelial-mesenchymal transition, KRAS signaling, myogenesis, and coagulation. Western blot results showed that the inhibition of IGFL2-AS1 could significantly reduce the N-cadherin, HIF1A and KRAS protein expression, yet increase the E-cadherin protein level. IGFL2-AS1 was also positively correlated with M0 macrophages, M2 macrophages, and neutrophils but negatively correlated with CD4+ memory $\mathrm{T}$ cells and CD8+ $\mathrm{T}$ cells.

Conclusion: IGFL1-AS1 could seriously worsen patient outcomes and facilitate COAD progression, thus serving as an independent tumor marker.

Keywords: IGFL2-AS1, IncRNA, colon cancer, immune

\section{Introduction}

Colorectal cancer $(\mathrm{CRC})$ is the third most prevalent cancer and the fourth leading cause of cancer-related deaths worldwide, where colon adenocarcinoma (COAD) is the primary subtype. ${ }^{1}$ Recently, CRC incidence and mortality have been steadily declining because of early detection and lifestyle change. ${ }^{2}$ From the 1970 s to 2012 , the five-year survival rate of CRC in all stages has increased from $51 \%$ to $66 \%{ }^{3}$ Nowadays, surgical resection combined with adjuvant therapy, such as radiotherapy, chemotherapy, and immunotherapy, remains the only curative option for $\mathrm{CRC}{ }^{4}$ However, with different therapy combinations, the long-term survival rate is still 
suboptimal for advanced-stage CRC. ${ }^{5}$ Therefore, new targeted therapies and markers of CRC need to be identified and developed urgently.

Long-chain non-coding RNA (lncRNAs) are transcripts of more than 200 nucleotides that are not translated into proteins, and most of them were formerly regarded as useless in the human genome. ${ }^{6}$ However, accumulated evidence over the last decade has indicated their crucial regulatory function in various diseases, such as cis-/transtranscriptional regulation, organization of nuclear domains, protein interaction, and RNA molecule regulation. ${ }^{7}$ Researchers have also extensively investigated the role of IncRNAs in cancers and found a tight relation between lncRNAs and tumor development. ${ }^{8}$ For example, He et al revealed that the lncRNA PCGEM1 is highly expressed in prostate cancer and could facilitate the proliferation of tumor cells through binding with miR-145. ${ }^{9}$ Gutschner et al demonstrated that the lncRNA MALAT1 is a prognosis marker and could promote tumor metastasis through a MALAT1 knockout model. ${ }^{10}$ In CRC, KCNQ1OT1 is associated with the response to fluoropyrimidine-based chemotherapy and could predict diseasefree survival in the early stages. ${ }^{11}$

Currently, the rapid development of bioinformatics and next-generation sequencing brings great convenience for relevant biological research. In parallel, the massive data generated provide comprehensive information on disease occurrence and development. In this study, we first identified IGFL2-AS1 as a candidate gene through a series of bioinformatics analysis. A high level of IGFL2-AS1 was found associated with worse clinical features and patient survival. In vitro experiments showed that the knockdown of IGFL2-AS1 could suppress the proliferation and invasion of COAD cells. Bioinformatics analysis showed that IGFL2-AS1 might affect the COAD tumor microenvironment.

\section{Methods}

\section{Acquisition of Public Data}

The bulk sequence data of colon cancer patients were obtained from TCGA portal sites (https://portal.gdc.can cer.gov/; TCGA-COAD), including 41 healthy samples and 473 tumor samples. ${ }^{12}$ The original ENSMBLE IDs were annotated using the latest reference file, GRCh38.gtf. The gene expression profile was normalized, and missing values were removed. Clinical information, including survival data, was downloaded in the form of "bcr xml" file and collated by Perl code. The differentially expressed lncRNAs (DELs) were identified using "limma package" with the threshold of $|\operatorname{logFC}|>1$ and $\mathrm{P}<0.05$.

\section{Tissue Specimens and Cell Lines}

Eight paired COAD tissue were collected from the Affiliated Hospital of YouJiang Medical University For Nationalities in accordance with the Declaration of Helsinki. All patients signed the informed consent before the operation, and the ethics committees approved the consent procedure. Colon cancer cell lines HCT116, HCT8, HCT129, and SW480 and normal colonic epithelial cell line NCM460 were purchased from iCell (Shanghai, China). All these cell lines were cultured under standard conditions $\left(37^{\circ} \mathrm{C}, 5 \% \mathrm{CO}^{2}\right)$.

\section{Quantitative Real-Time PCR (qRT-PCR)}

Total RNA of tissues and cell lines was extracted using the RNA simple Total RNA kit (TIANGEN, Beijing, China). cDNA was reversely transcribed using the Fast Quant RT kit (TIANGEN, Beijing, China). The mRNA level of target genes was detected in 96-well plates by using Super Real PreMix Plus SYBR Green (TIANGEN, Beijing, China). The primers used were as follows: IGFL2-AS1, forward primer, 5'AGCCTATTTCCAGACAACT-3', reverse primer, 5'AGAATCAACGACCTCTACAT-3'; GAS1RR, forward primer, 5'-TGGCATTTGGCTAGGTTC-3', reverse primer, 5'AACAGGGTTGGGAGTGGGTATTTGC-3'; AC011352.3, forward primer, 5'-AGGCTATTGAGGCAGATT-3', reverse primer, 5'-TTCCTGTCCAGCAAAGAT-3'; HOTAIR, forward primer, 5'-GGCAAATGTCAGAGGGTT-3', reverse primer, 5'-CTTAAATTGGGCTGGGTC-3'; AC093895.1, forward primer, 5'-CCCCAGTCTTGTGCCTAT-3', reverse primer, 5'-CACCACGAAGCGAGATAA-3'; AC007785.1, forward primer, 5'-AAACTGACCCTCCTTCTT-3', reverse primer, 5'-CTCCTTGGCTCCTATACTC-3'; AC104809.2, forward primer, 5'-TCTGCTGAAAGACCCGTTAT-3', reverse primer, 5'-TCGGTGGAGTGATTGGTTAG-3'.

\section{Western Blot}

Total protein of control and IGFL2-AS1 knockdown cells were collected with RIPA lysis buffer at $4{ }^{\circ} \mathrm{C}$ for 1.5 h. Western blot was performed using $10 \%$ SDSpolyacrylamide gel electrophoresis (PAGE) to semiquantitatively show protein level. The primary antibody used were all purchased from proteintech (Proteintech Group, Inc), including anti-E-cadherin, anti-N-cadherin, 
anti-HIF1A, anti-KRAS. The GAPDH antibody were obtained from laboratory stocks.

\section{Immunofluorescence}

The tissue of high and low IGFL2-AS1 expression patients was frozen and sectioned at a thickness of $5 \mu \mathrm{m}$. These tissue section slides were fixed, washed, and blocked with PBS containing BSA and FBS. CD206 primary antibody was purchased from proteintech (Proteintech Group, Inc). The second antibody was goat anti-mouse IgG heavy and light chain. After the immunofluorescence, cells were photoed using a immunofluorescence microscopy.

\section{Cell Transfection}

IGFL2-AS1 small interfering RNAs (siRNA; 5'GCCCAGATCAACAGAATCA-3') and negative control (NC) siRNA were purchased from Thermo Fisher Scientific. Lipofectamine 2000 (Invitrogen) was used to perform the transfection of siRNA-LINC00662 and siRNA-NC. The transfection efficiency was measured by qRT-PCR assay.

\section{Transwell Invasion and Migration Assays}

Transwell assay was performed using 24 transwell chambers (Corning) to assess the invasion and migration of cancer cells. Cells at a density of $4 \times 10^{3}$ were seeded into each upper chamber with a serum-free conditioned medium. Bottom chambers were filled with a culture medium containing $10 \%$ fetal bovine serum. After $24 \mathrm{~h}$, the cells migrated to the lower chamber were stained with $0.1 \%$ crystal violet.

\section{Wound-Healing Assay}

Cells were plated into six-well plates to $90 \%$ confluence with serum-free conditioned medium. Cell gaps were scratched in each well using a $100 \mu \mathrm{L}$ tip set to indicate cell boundaries at 0 and $24 \mathrm{~h}$.

\section{Cell Proliferation Assays}

CCK8 and colony formation assays were used to test cell proliferation. CCK8 assay was conducted using a CCK8 Kit in accordance with the manufacturer's protocol (Dojindo, Shanghai, China). Colony formation assay was performed with cells seeded into six-well plates with 300 cell per well. The medium was replaced every 4 days. Cell counts were conducted after 14 days.

\section{Flow Cytometry}

Cell apoptosis was evaluated by flow cytometry. In brief, the cells were resuspended with phosphate-buffered saline and stained with Annexin V/propidium iodide at room temperature for $20 \mathrm{~min}$ (Invitrogen, Inc., Carlsbad, CA, USA). The resulting fluorescence was detected using flow cytometry.

\section{Pathway Enrichment Analysis}

Gene set enrichment analysis (GSEA) was performed between patients with high and low IGFL2-AS1 to explore the underlying biological pathway of IGFL2-AS1. ${ }^{13}$ The gene set database was "Hallmark gene set v7.2," the number of permutations was 1000 , the metric for ranking genes was "Signal2Noise," the normalization mode was "meandiv," and the randomization mode was "no_balance." GO analysis was conducted using the "Clusterprofiler" package in $\mathrm{R}$ software, which included three modules, namely, biological process (BP), cellular component (CC), and molecular function $(\mathrm{MF})$.

\section{Immune Infiltration Analysis}

CIBERSORT algorithms were used to calculate the proportion of 22 immune cells in tumor microenvironment, a deconvolution algorithm designed to quantify the abundance of specific cell types based on a standardized gene expression profile. The expression profile was processed with the following steps before CIBERSORT analysis: deletion of normal samples, removal of low abundance genes, and data correlation. In brief, for the parameters of CIBERSORT, the number of permutations was set as "100," the kappa was set as "999," and the q-value was set as " 0.3 ". The reference file and data source were uploaded in https://figshare.com/articles/dataset/CIBERSORT/ 14741916 .

\section{Statistical Analysis}

All analyses were performed in R software v4.0, SPSS v13.0 and GraphPad Prism 8. The P-value used in the analysis was two-sided, and $<0.05$ was regarded as statistically significant. Student paired $t$-test was used to compare the level of variables between two groups. Wilcox test was used to analyze statistical significance between multiple groups. All experiments were repeated three times. Data are presented as mean \pm standard deviation (SD). 


\section{Results}

\section{Identification of Differentially Expressed IncRNAs}

Differential analysis showed that 2443 DELs were identified between COAD and normal tissue, of which 1526 were upregulated and 921 were downregulated (Figure 1A). Similarly, 46 upregulated and 14 downregulated lncRNAs were differentially expressed between the metastatic and non-metastatic groups (Figure 1B); 149 upregulated and eight downregulated DELs were identified between the high (T3 and T4) and low $\mathrm{T}$ (T1 and T2) classification groups (Figure 1C). We next intersected these DELs and found seven shared lncRNAs, namely, IGFL2-AS1, GAS1RR, AC011352.3, HOTAIR, AC093895.1, AC007785.1, and AC104809.2 (Figure 1D and Table 1). Then, we conduct survival analysis to explore the underlying effect of these seven IncRNAs on patients survival. KaplanMeier survival curve indicated that the patients with high levels of the seven IncRNAs were associated with worse overall survival (OS) (Figure 1E-K).

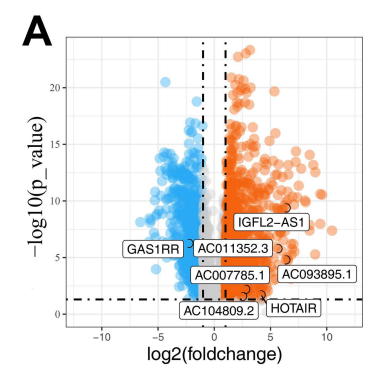

E
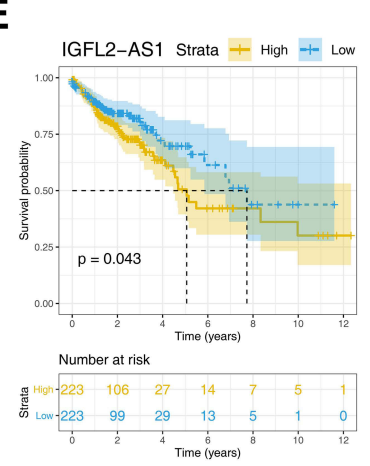

B

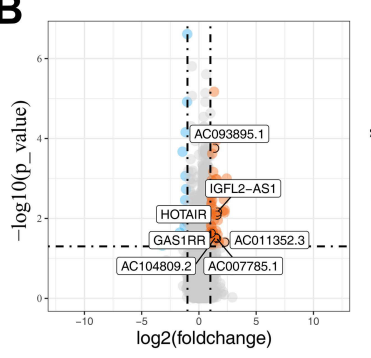

$\mathbf{F}$
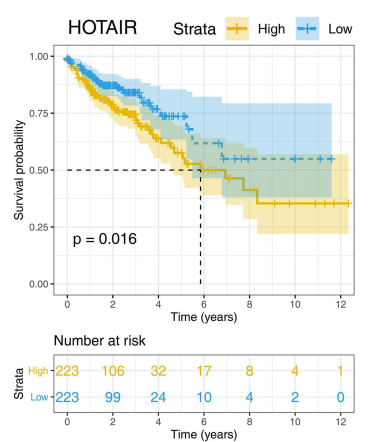

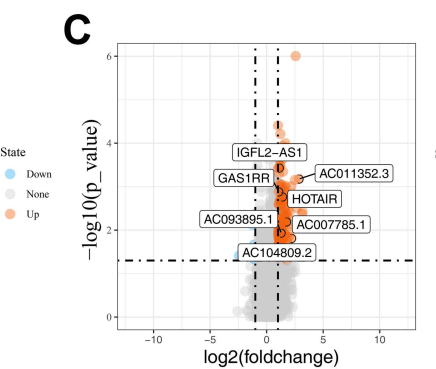

G
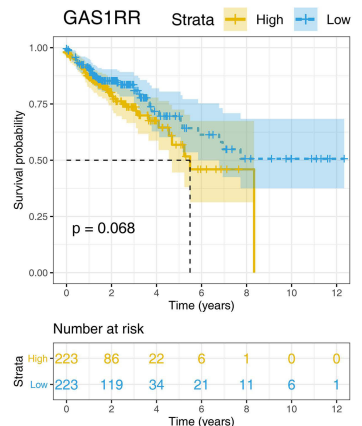

D

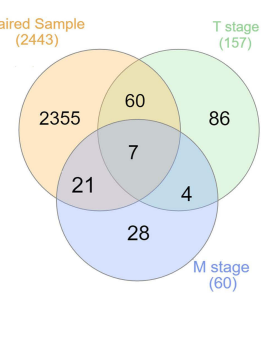

H
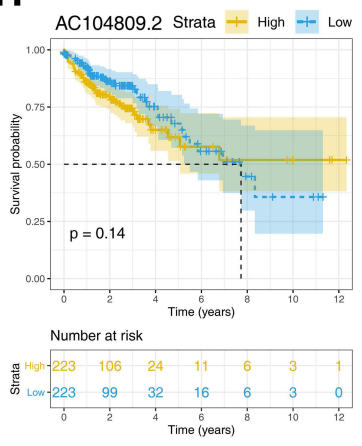
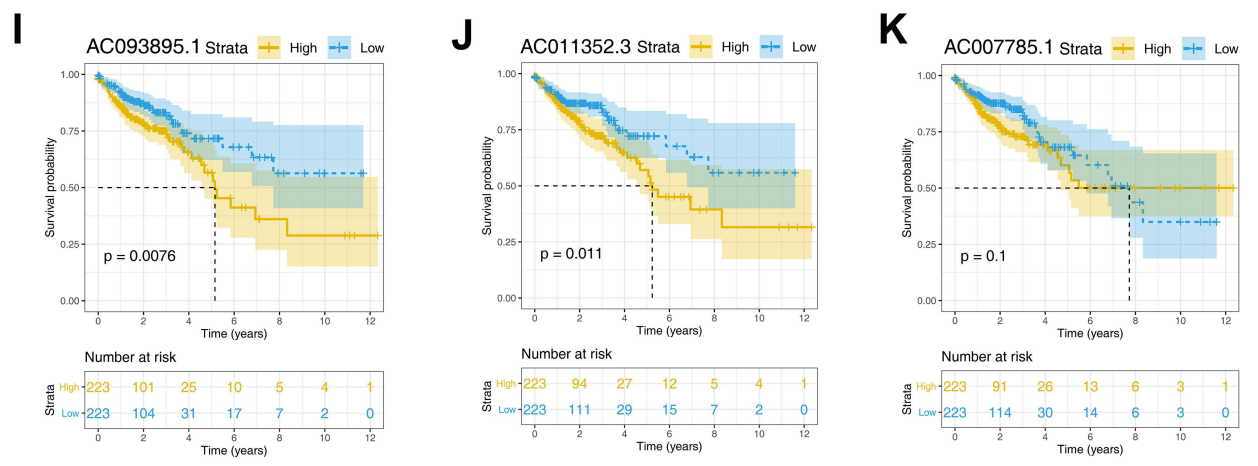

Figure I Identification of DELs in different groups.

Notes: (A) The volcano plot of DELs between tumor and normal tissue with the threshold of $|\operatorname{logFC}|>\mid$ and P.value $<0.05$ (I526 up-regulated and 92 I down-regulated); (B) The volcano plot of DELs between metastatic and non-metastatic groups with the threshold of $|\operatorname{logFC}|>\mid$ and P.value $<0.05$ (46 up-regulated and I4 down-regulated); (C) The volcano plot of DELs between high and low T classification groups with the threshold of $|\operatorname{logFC}|>\mid$ and P.value $<0.05$ (I49 up-regulated and 8 down-regulated); (D) The venn plot of intersected IncRNAs between three groups mentioned above; (E-K) The Kaplan-Meier survival curves of seven intersected IncRNAs, IGFL2-ASI, GASIRR, AC0II352.3, HOTAIR, AC093895.I, AC007785.I and ACI04809.2.

Abbreviation: DELs, differentially expressed IncRNAs. 
Table I The Expression Level of Identified Seven IncRNAs in Tumor and Normal Tissue

\begin{tabular}{|l|c|c|c|c|c|}
\hline LncRNA & conMean & treatMean & logFC & pValue & fdr \\
\hline IGFL2-ASI & 0.03 & 2.17 & 6.38 & $<0.01$ & $<0.01$ \\
GASIRR & 0.42 & 0.09 & -2.25 & $<0.01$ & $<0.01$ \\
ACOII352.3 & $<0.01$ & 0.25 & 5.65 & $<0.01$ & $<0.01$ \\
HOTAIR & 0.07 & 1.22 & 4.16 & 0.02 & 0.03 \\
AC093895.I & 0.01 & 0.42 & 6.36 & 0.01 & $<0.01$ \\
AC007785.I & 0.09 & 0.62 & 2.79 & 0.01 & 0.01 \\
ACI04809.2 & 0.06 & 0.33 & 2.51 & 0.03 & 0.04 \\
\hline
\end{tabular}

\section{Clinical Correlation of IGFL2-ASI}

The IncRNA IGFL2-AS1 had the highest expression level in COAD tissue and therefore was used in further study (treatMean $=2.17 ; \operatorname{logFC}=6.38$; Table 1). Also, the relative expression level of the other six lncRNAs was assessed with qRT-PCR and shown in Figure S1. Clinical correlation analysis of IGFL2-AS1 showed no significant difference in different age and gender groups (Figure 2A and B). Notably, a higher level of IGFL2-AS1 was associated with worse clinical features, including stage, $\mathrm{T}$ classification, $\mathrm{N}$ classification, and $\mathrm{M}$ classification (Figure 2C-F).

\section{IGFL2-ASI is Highly Expressed in COAD Tissue and Cell Lines}

Next, we performed a qRT-PCR analysis based on the total RNA extracted from eight paired tumor and normal tissue. The result revealed a higher IGFL2-AS1 mRNA level in COAD tissue than in healthy colon epithelial tissues (Figure 3A). In parallel, a high IGFL2-AS1 level was also observed in COAD cell lines, including HCT116, HCT8, HCT129, and SW480 (Figure 3B). To explore the biological role of IGFL2-AS1 in COAD, we performed cell transfection to interfere with endogenous IGFL2-AS1 expression. In
A

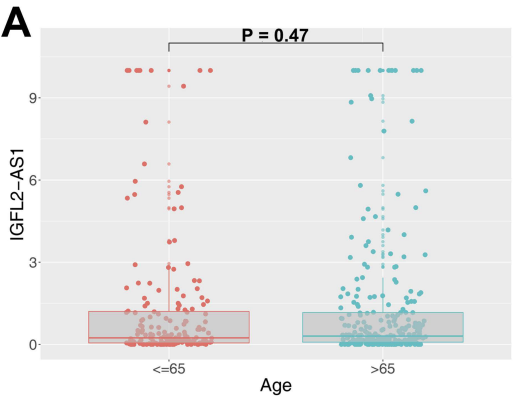

D

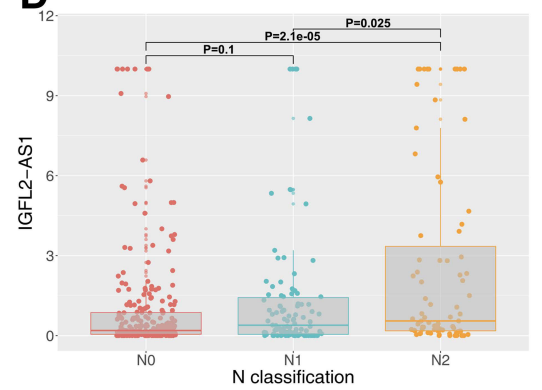

B

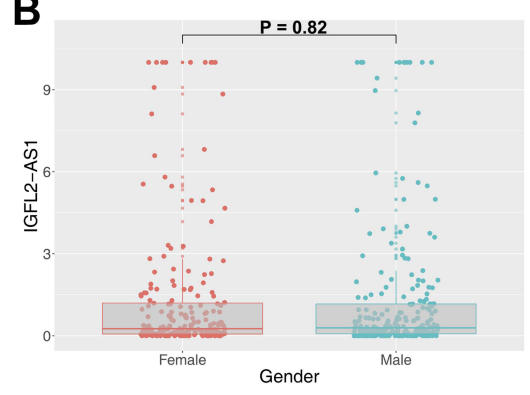

E

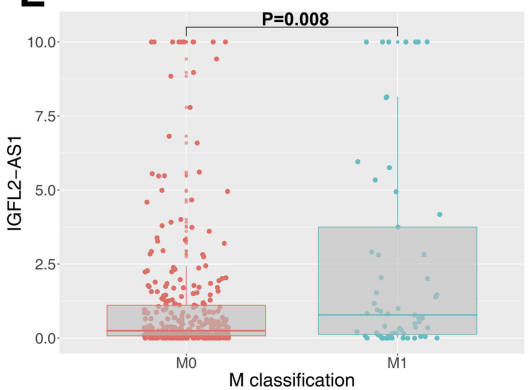

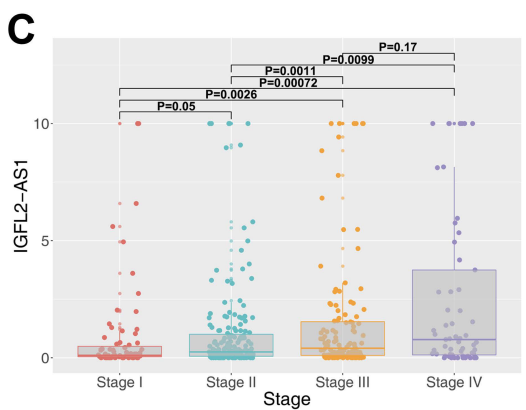

$\mathbf{F}$

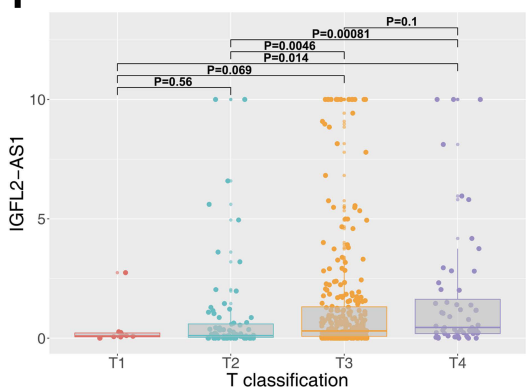

Figure 2 Clinical correlation of IGFL2-ASI.

Notes: (A) The expression value of IGFL2-ASI in different age groups ( $\leq 65$ and $>65$ ); (B) The expression value of IGFL2-ASI in male and female groups; (C) The expression value of IGFL2-ASI in different clinical-stage group (Stage I, Stage II, Stage III and Stage IV); (D) The expression value of IGFL2-ASI in different N classification groups (N0, NI and N2); (E) The expression value of IGFL2-ASI in different M classification groups (M0 and MI); (F) The expression value of IGFL2-ASI in different T classification groups (TI, T2, T3 and T4). 
A

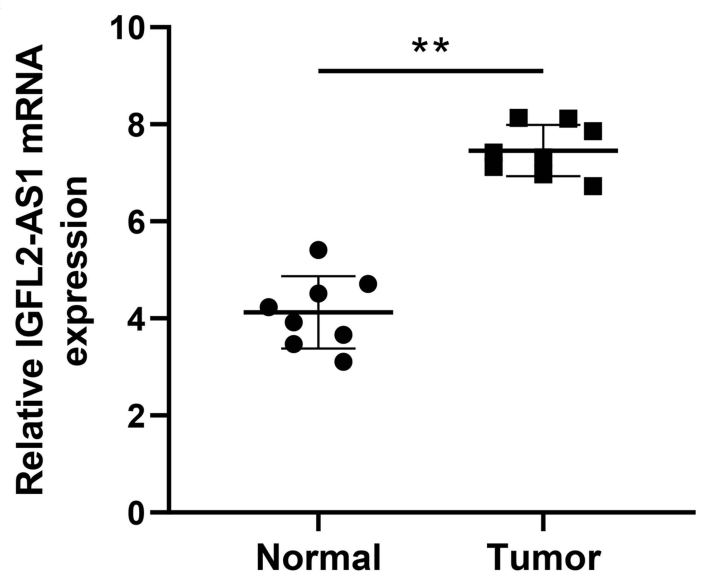

B

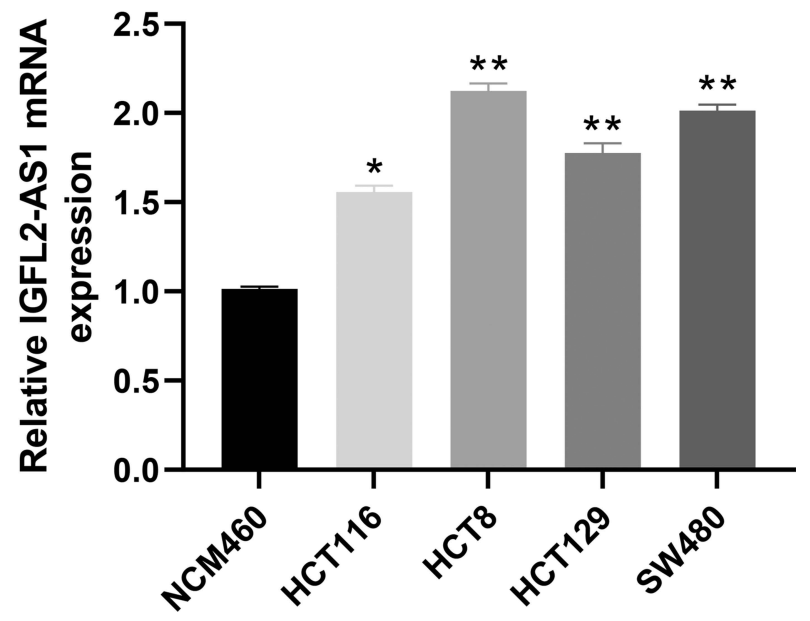

Figure 3 The IncRNA IGFL2-ASI is highly expressed in COAD tumor tissue and cells.

Notes: (A) IGFL2-ASI is highly expressed in COAD tissue (eight paired tumor and normal tissue), *P<0.05, **P<0.0I; (B) IGFL2-ASI is highly expressed in COAD cell lines, HCTII6, HCT8, HCTI29 and SW480, *P<0.05, **P<0.0I.

addition, the transfection efficiency of IGFL2-AS1 was validated by qRT-PCR (Figure 4A and B).

\section{Knockdown of IGFL2-ASI Inhibits the Invasion and Proliferation of COAD Cells}

The cell phenotype experiments were then performed to assess the impact of IGFL2-AS1 on cell malignant biological behaviors, including transwell, wound-healing, colony formation and CCK8 assay. The transwell assay showed that IGFL2-AS1 knockdown could significantly decrease the number of migration and invasion cells, indicating that IGFL2-AS1 promotes tumor metastasis (Figure 4C and D). The wound-healing assay also validated this phenotype, in which a prominent wound healing area was observed in the si-IGFL2-AS1 group (Figure 4E). Colony formation assay suggested that IGFL2-AS1 knockdown reduced the colony number in HCT8 and SE480 cell lines (Figure 5A). Meanwhile, we found a higher apoptosis rate in the si-IGFL2-AS1 group than in the si-NC group (Figure 5B). CCK8 assay also demonstrated that IGFL2AS1 knockdown can significantly inhibit the proliferation of COAD cells (Figure 5C).

\section{Pathway Enrichment Analysis of IGFL2-AS I}

We conducted GSEA analysis to understand the underlying biological function of IGFL2-AS1 in COAD. Based on the Hallmark gene set, in the high IGFL2-AS1 phenotype, the top five upregulated oncogenic pathways were "angiogenesis," "epithelial-mesenchymal transition," "KRAS signaling," "myogenesis," and "coagulation" (Figure 6A). GO analysis showed that the main enriched terms for BP were "cell-cell adhesion," "epidermis development," and "Hormone metabolic process." For CC, the IGFL2-AS1 was markedly enriched in "cornified envelope," "Golgi lumen," and "DNA packaging complex." Changes in CC were prominently enriched in "extracellular matrix structural," "monooxygenase activity," and "metallopeptidase activity" (Figure 6B). To validate the underlying effect of IGFL2-AS1 on the angiogenesis, EMT and KRAS signaling, we performed Western blot assay in control and IGFL2-AS1 knockdown cells. The result showed that the inhibition of IGFL2-AS1 could significantly reduce the N-cadherin, HIF1A and KRAS protein expression, yet increase the E-cadherin protein level (Figure 6C).

\section{Immune Infiltration Analysis}

Considering the pivotal role of immune cells in tumor microenvironment, we explored the association of IGFL2-AS1 with 22 immune cells by using the CIBERSORT algorithm. Results revealed that IGFL2-AS1 was positively correlated with M0 macrophages, M2 macrophages, and neutrophils but negatively correlated with CD4+ memory $\mathrm{T}$ cells and CD8+ T cells (Figure 7A).

The impact of IGFL2-AS1 on M2 macrophages was selected to further validation. Furthermore, in immunofluorescence slides, the tumor tissue with high IGFL2AS1 expression showed a prominent high M2 macrophage 

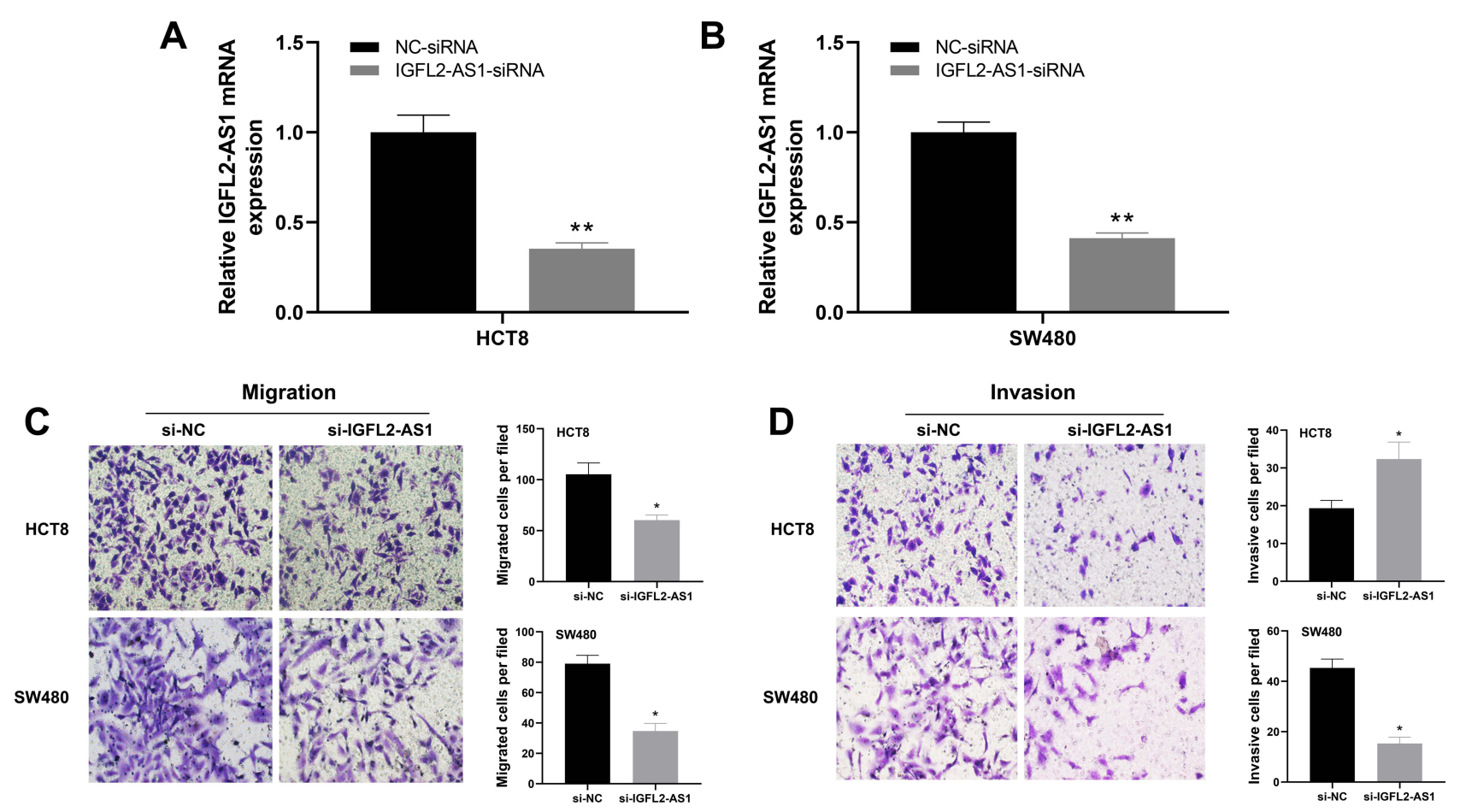

E

si-NC
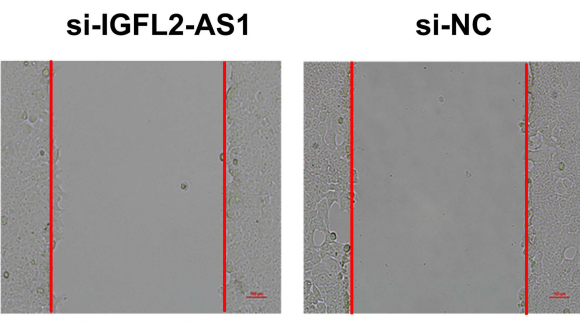

si-IGFL2-AS1
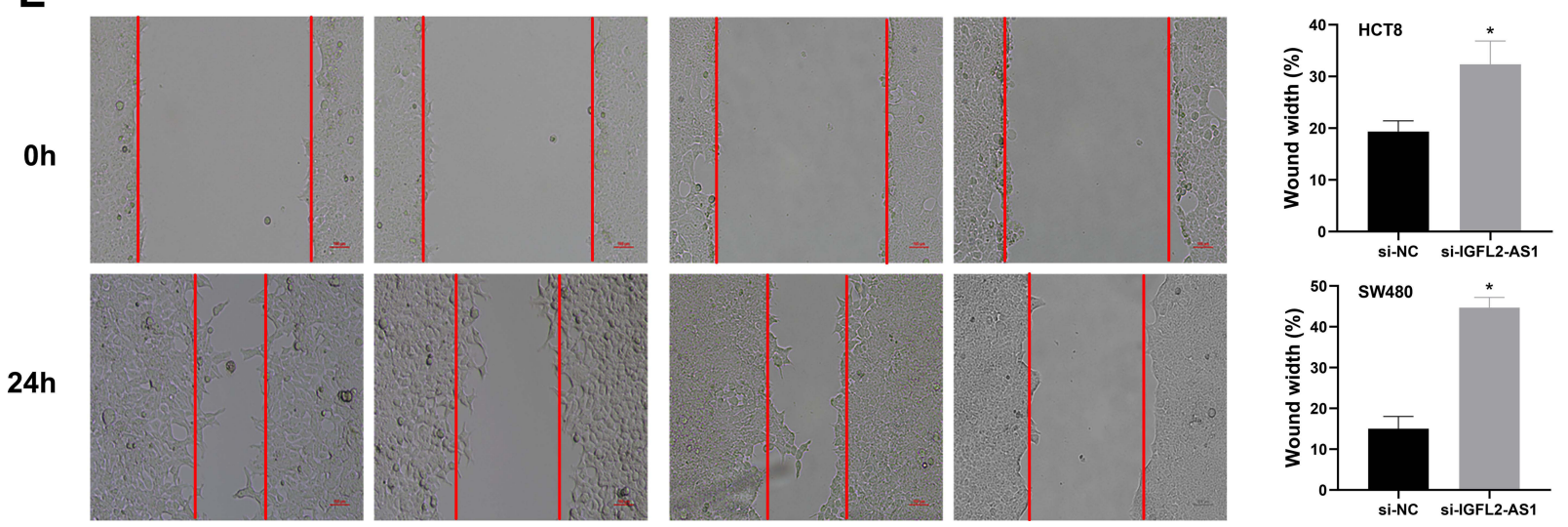

24h
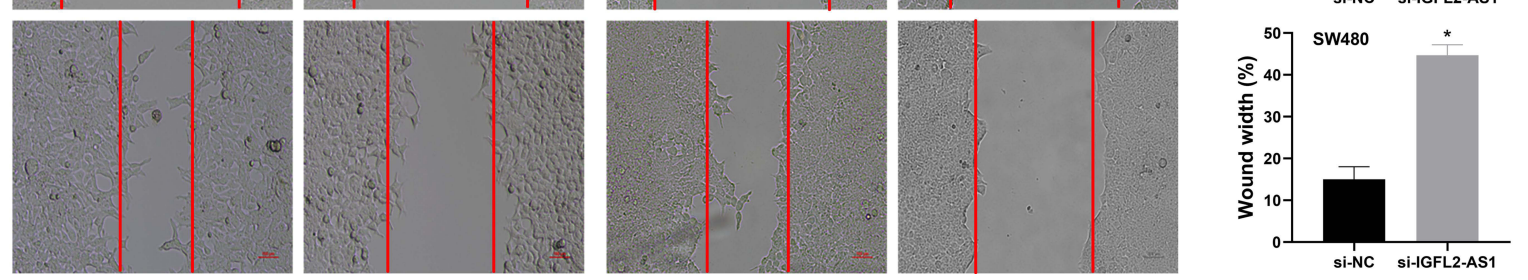

Figure 4 IGFL2-ASI promotes migration and invasion of COAD cells.

Notes: (A and B) RT-qPCR of IGFL2-ASI in si-NC and si-IGFL2-ASI groups, ${ }^{* P}<0.05, * * P<0.01$; (C and D) Downregulation of IGFL2-ASI reduced the number of migration and invasion cells in the transwell assay (HCT8 and SW480 cell lines), $* \mathrm{P}<0.05, * * \mathrm{P}<0.0 \mathrm{I} ;$ (E) Wound-healing assay revealed that downregulation of IGFL2-ASI significantly reduced the migration rate ( $\mathrm{HCT} 8$ and $\mathrm{SW} 480$ cell lines), $* \mathrm{P}<0.05, * * \mathrm{P}<0.01$.

infiltration compared with the tissue with low IGFL2-AS1 (Figure 7B).

\section{Discussion}

As the three most common malignant tumors globally, COAD is still the major cause of cancer death worldwide. Despite remarkable advancement in COAD therapy, the five-year survival rate of later stages is only approximately $10 \%$ because of the recurrent disease and chemotherapeutic drug resistance. Therefore, searching for novel cancer and therapeutic markers for COAD is crucial.
To the best of our knowledge, this study is the first to analyze the role of IGFL2-AS1 in COAD to the best of our knowledge. We first identified lncRNA IGFL2-AS1 for its underlying cancer-promoting role in patients with COAD. We also first validated the high expression level of IGFL2AS1 in COAD tissue and cell lines through a qRT-PCR assay. In vitro experiment demonstrated that IGFL2-AS1 can significantly facilitate the proliferation and invasion but inhibit the apoptosis of COAD cells. We further explored the biological pathway and immune infiltration difference between patients with high and low IGFL2-AS1 expression levels. 
A
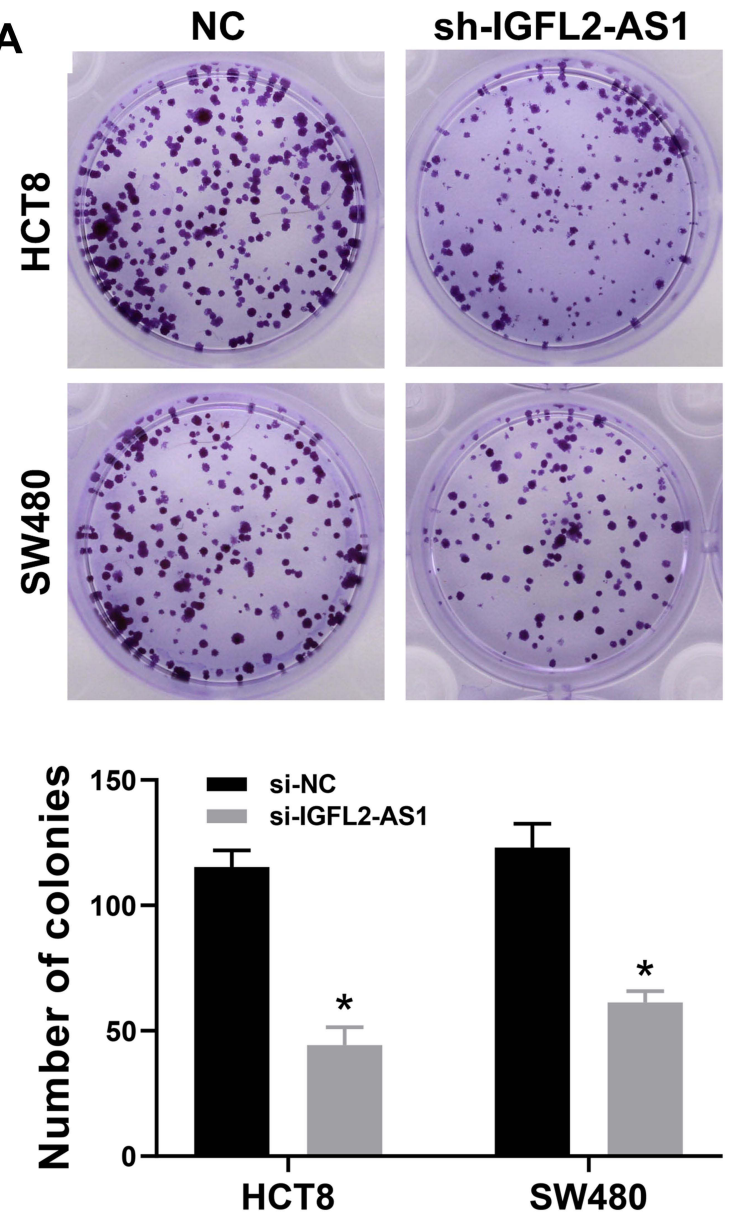

C

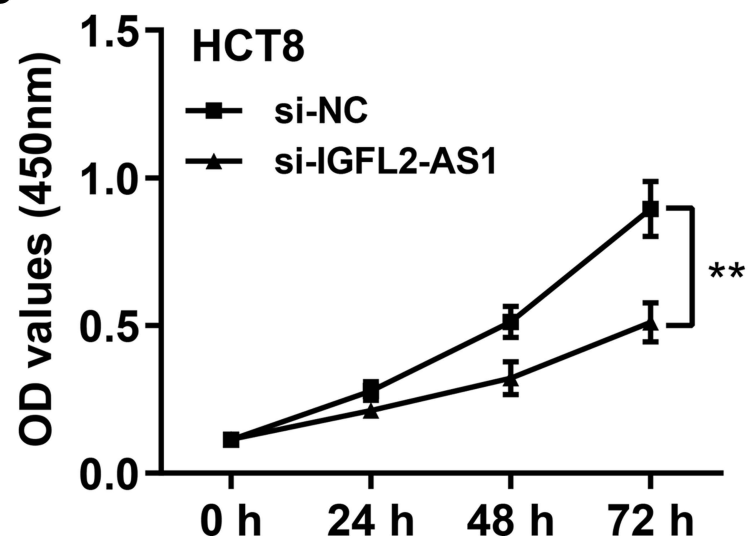

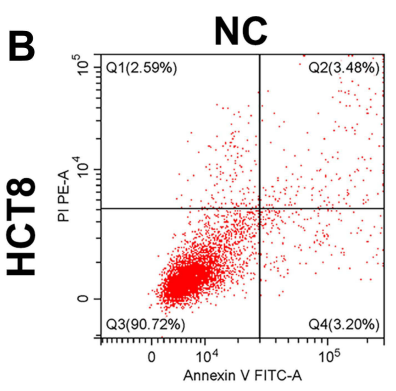

sh-IGFL2-AS1
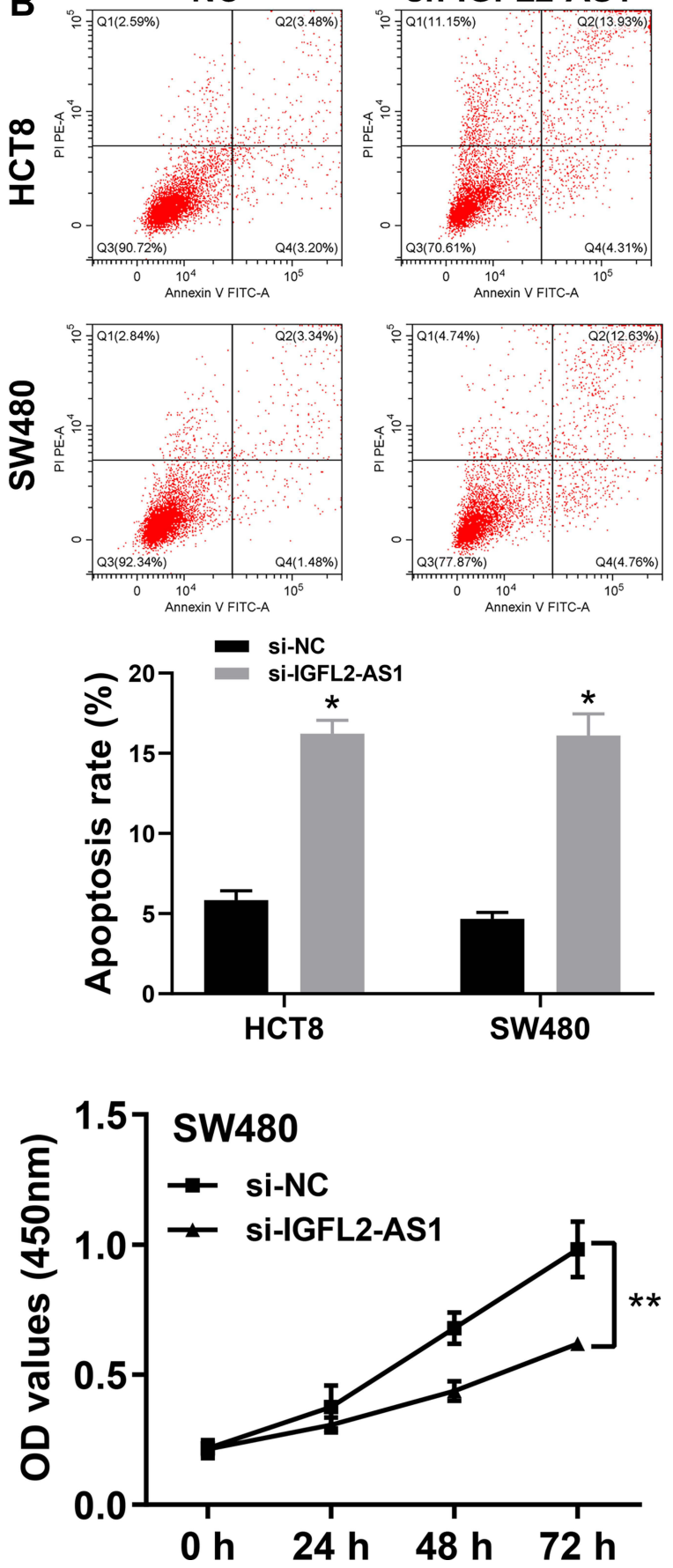

Figure 5 IGFL2-ASI promotes proliferation of COAD cells.

Notes: (A) Downregulation of endogenous IGFL2-ASI reduced the mean colony number in the colony formation assay (HCT8 and SW480 cell lines), * $=\mathrm{P}<0.05$, **P $<0.0 \mathrm{I}$; (B) Flow cytometry showed that the downregulation of endogenous IGFL2-ASI significantly increased cell apoptosis (HCT8 and SW480 cell lines), $* \mathrm{P}<0.05$, $* * \mathrm{P}<0.0 \mathrm{I}$. (C) CCK8 assays revealed that downregulation of endogenous IGFL2-ASI significantly reduced the cell viability (HCT8 and SW480 cell lines).

Nowadays, lncRNAs are widely involved in cancer initiation and progression, especially in epigenetic regulation, protein interaction, and RNA metabolism. For example, Yue et al found that IncRNA CYTOR could form a positive feed-forward loop with $\mathrm{Wnt} / \beta$-Catenin signaling and thus promote the metastasis of COAD. ${ }^{14}$ Cheng et al demonstrated that IncRNA LINC00662 could regulate CLDN8/IL22 co-expression through binding with miR140-5p, which might activate the ERK signaling pathway and promote tumor development. ${ }^{15}$ In gastric cancer, Feng et al suggested that IncRNA HOXA-AS3 could activate NF- $\kappa \mathrm{B}$ signaling with a competitive endogenous RNA 

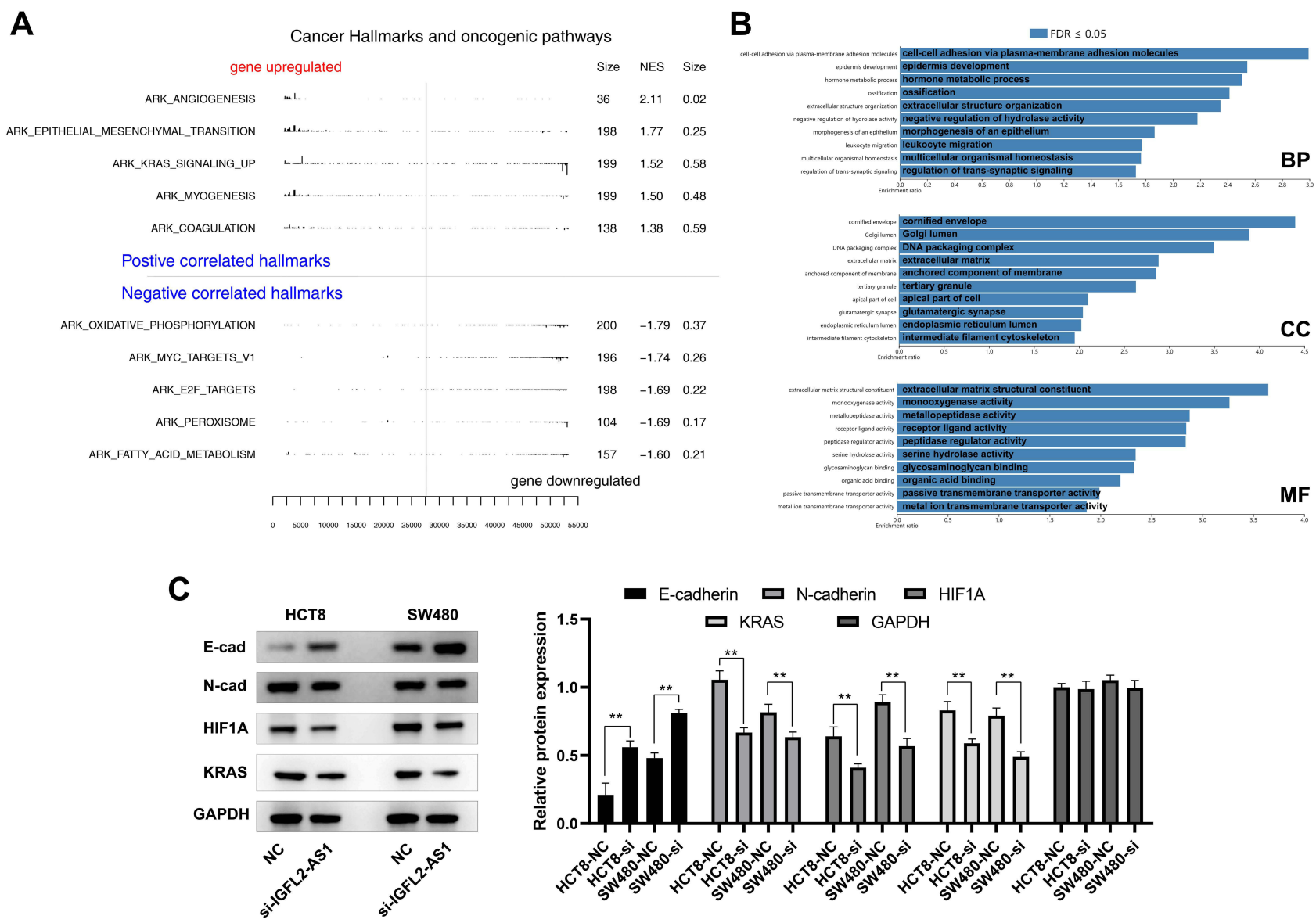

Figure 6 GSEA pathway enrichment analysis of IGFL2-ASI.

Notes: (A) GSEA analysis to explore the biological pathway IGFL2-ASI involved in (the upper part were the pathways up-regulated in the IGFL2-ASI high expression patients, the lower part were the pathways down-regulated in the IGFL2-ASI high expression patients); (B) GO analysis of IGFL2-ASI with FDR $\leq 0.05$ (three part, BP, biological process; CC, cellular component and MF, molecular function); (C) Western blot of E-cad, N-Cad, HIFIA, KRAS and GAPDH in control and IGFL2-ASI knockdown cells, $* \mathrm{P}<0.05, * * \mathrm{P}<0.01$.

Abbreviations: GSEA, gene set enrichment analysis; GO, gene ontology.

(ceRNA) mechanism (miR-29a-3p/LT $\beta R) .{ }^{16}$ In another aspect, the lncRNA MALAT1 is up-regulated in multiple types of malignant tumors and could interact with the SR protein family of splicing regulators. ${ }^{17}$ Meanwhile, Ma et al found that IGFL2-AS1 could promote gastric cancer development through binding to miR-802 with a ceRNA mechanism. ${ }^{18}$ The present study showed that IGFL2-AS1 is highly expressed in COAD and could promote tumor development. Moreover, patients with high IGFL2-AS1 expression appear to have a poor prognosis, indicating that IGFL2-AS1 might be a novel tumor marker in patients with COAD.

We conducted GSEA analysis to explore the biological pathway of IGFL2-AS1. Results showed that the top five activated pathways were angiogenesis, epithelialmesenchymal transition (EMT), KRAS signaling, myogenesis, and coagulation. The chaotic vascular system in the local microenvironment is a character of malignant tumors. $^{19}$ The high permeability of upstream vascular could lead to a low blood perfusion level because of blood and oxygen loss. This hypoxic microenvironment favors the colonies that are more adapted to harsh environments and thus enhances the proliferation and metastatic potential. $^{20}$ During EMT, epithelial cells are transdifferentiated into motile mesenchymal cells, which plays a vital role in cancer development. ${ }^{21}$ For instance, Feng et al indicated that CDC42EP3 could facilitate the malignant phenotype of colorectal cancer cells by regulating EMT. ${ }^{22}$ In addition, as a common mutant oncogene in carcinoma, KRAS plays an essential role in tumor progression. Buscail et al comprehensively reviewed the role of KRAS mutation in pancreatic cancer and found that the existence of KRAS mutant in serum and plasma is associated with a worse prognosis of pancreatic cancer. ${ }^{23}$ Lal 

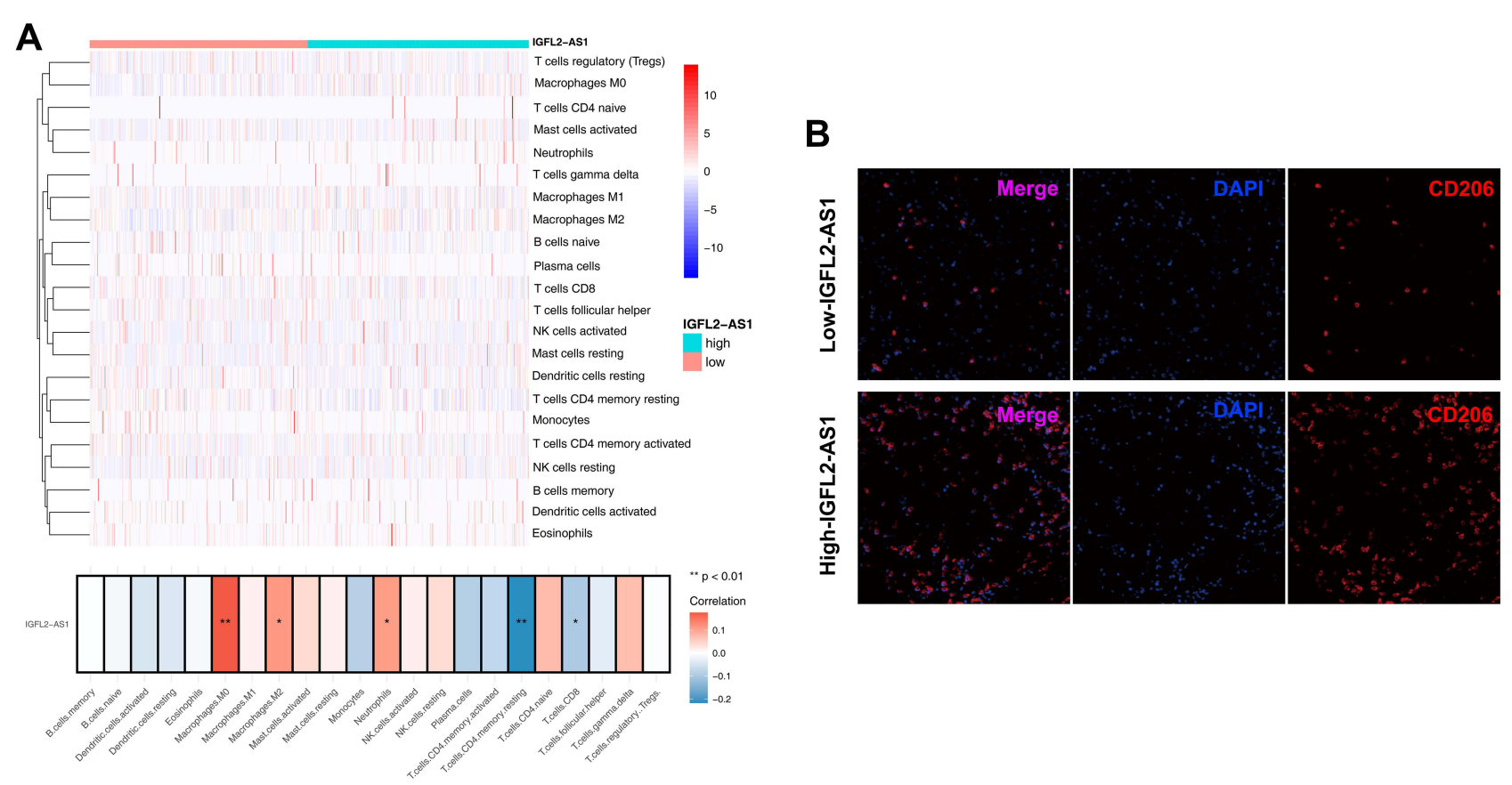

Figure 7 Immune infiltration analysis performed by CIBERSORT algorithms in high and low IGFL2-ASI group.

Notes: (A) The upper part is the heatmap overview of immune cell in the high and low IGFL2-ASI patients; the lower part showed that the IGFL2-ASI was positively correlated with M0 macrophages, M2 macrophages and neutrophils, while negatively correlated with CD4+ memory T cells and CD8+ T cells, *P<0.05, **P<0.0I; (B) Representative immunofluorescence image indicated different CD206 level in high and low IGFL2-ASI tissue.

et al revealed that KRAS mutation in COAD decreases the infiltration of neutrophils and cytotoxic cells, resulting in advanced disease progression. ${ }^{24}$

The heterogeneity of tumor microenvironment affects the progression of tumor cells significantly. ${ }^{12}$ We subsequently performed immune infiltration analysis to explore the difference in tumor immune microenvironment between patients with high and low IGFL2-AS1 expression levels. Results showed that IGFL2-AS1 increased the contents of M0 macrophages, M2 macrophages, and neutrophils but decreased the contents of CD4 memory T cells and CD8 T cells. In general, M0 macrophages could differentiate into M1 and M2 macrophages under different induced conditions. Zhang et al found that apoptotic SKOV3 cells could stimulate M0 macrophages to differentiate into M2 macrophages by activating the ERK signaling pathway, thus promoting the proliferation and migration of ovarian cancer cells. ${ }^{25}$ Neutrophils are involved in different tumor development stages, including tumor initiation, proliferation, and metastasis. ${ }^{26}$ Commonly, the reactive oxygen species, reactive nitrogen species, or proteases released by neutrophils could induce angiogenesis and promote tumor metastasis. ${ }^{27}$ Furthermore, the suppression of neutrophils on the immune system could facilitate tumor proliferation. For example, the anti-tumor effect mediated by CD8+ $\mathrm{T}$ lymphocytes can be suppressed by small molecules released by neutrophils. ${ }^{28,29} \mathrm{CD} 8+\mathrm{T}$ cells are the preferred choice targeting cancer therapy. The systemic review performed by Farhood et al concluded that effector CD8+ cytotoxic $\mathrm{T}$ lymphocytes can infiltrate the core site of the tumor tissue and exert a killing effect. ${ }^{30}$

Despite our findings, this study has several limitations. First, the public data included in our analysis were predominantly western people. Thus, whether or not the research results hold true for other populations needs to be further verified. Second, as clinical features records of some patients are incomplete, our conclusions affected by this issue may exhibit potential bias. Third, the in-depth mechanism by which IGFL2-AS1 promotes tumor development still needs to be validated through in vitro and in vivo experiments. Therefore, based on the direction provided in this analysis, we will focus on the underlying mechanism of IGFL2-AS1 in COAD through related biological experiments and cohort validation.

\section{Conclusion}

Considering its close relation to clinical features and patient survival, we first identified IGFL2-AS1 as a target gene through a series of bioinformatics analyses. 
In vitro experiments showed that IGFL2-AS1 was highly expressed in COAD tissue and could promote the proliferation and invasion of cancer cells (HCT8 and SW480). GSEA analysis indicated that the top three biological pathways involving IGFL2-AS1 were angiogenesis, EMT, and KRAS signaling. CIBERSORT analysis showed that IGFL2-AS1 was positively correlated with M0 macrophages, M2 macrophages, and neutrophils but negatively correlated with CD4+ memory T cells and CD8+ T cells.

\section{Funding}

This work was supported by Special Funding for Guangxi Special Experts (GRCT2019-13); Guangxi Medical Highlevel Leading Talents Training “139” Project (GWKJ201822).

\section{Disclosure}

The authors report no conflicts of interest in this work.

\section{References}

1. Siegel RL, Miller KD, Fuchs HE, Jemal A. Cancer statistics, 2021. CA Cancer J Clin. 2021;71(1):7-33.

2. Edwards BK, Ward E, Kohler BA, et al. Annual report to the nation on the status of cancer, 1975-2006, featuring colorectal cancer trends and impact of interventions (risk factors, screening, and treatment) to reduce future rates. Cancer. 2010;116(3):544-573. doi:10.1002/ cncr. 24760

3. Siegel R, Desantis C, Jemal A. Colorectal cancer statistics, 2014. $C A$ Cancer J Clin. 2014;64(2):104-117.

4. Thanikachalam K, Khan G. Colorectal cancer and nutrition. Nutrients. 2019;11(1):164. doi:10.3390/nu11010164

5. Miles A, van Duijnhoven F, McQueen A, Oliphant R. Colorectal cancer: advances in prevention and early detection. Biomed Res Int 2015;2015:518068. doi:10.1155/2015/518068

6. Fu D, Shi Y, Liu JB, et al. Targeting long non-coding RNA to therapeutically regulate gene expression in cancer. Mol Ther Nucleic Acids. 2020;21:712-724. doi:10.1016/j.omtn.2020.07.005

7. Ulitsky I, Bartel DP. lincRNAs: genomics, evolution, and mechanisms. Cell. 2013;154(1):26-46. doi:10.1016/j. cell.2013.06.020

8. Bhan A, Mandal SS. Long noncoding RNAs: emerging stars in gene regulation, epigenetics and human disease. ChemMedChem. 2014;9 (9):1932-1956. doi:10.1002/cmdc.201300534

9. He JH, Zhang JZ, Han ZP, Wang L, Lv YB, Li YG. Reciprocal regulation of PCGEM1 and miR-145 promote proliferation of LNCaP prostate cancer cells. J Exp Clin Cancer Res. 2014;33 (1):72. doi:10.1186/s13046-014-0072-y

10. Gutschner T, Hämmerle M, Eissmann M, et al. The noncoding RNA MALAT1 is a critical regulator of the metastasis phenotype of lung cancer cells. Cancer Res. 2013;73(3):1180-1189. doi:10.1158/00085472.CAN-12-2850

11. Lapucci A, Perrone G, Di Paolo A, et al. PNN and KCNQ1OT1 can predict the efficacy of adjuvant fluoropyrimidine-based chemotherapy in colorectal cancer patients. Oncol Res. 2021;28(6):631-644. doi:10.3727/096504020X16056983169118

12. Wang Z, Jensen MA, Zenklusen JC. A practical guide to the cancer genome atlas (TCGA). Methods Mol Biol. 2016;1418:111-141.
13. Subramanian A, Tamayo P, Mootha VK, et al. Gene set enrichment analysis: a knowledge-based approach for interpreting genome-wide expression profiles. Proc Natl Acad Sci USA. 2005;102 (43):15545-15550. doi:10.1073/pnas.0506580102

14. Yue B, Liu C, Sun H, et al. A positive feed-forward loop between LncRNA-CYTOR and Wnt/ $\beta$-catenin signaling promotes metastasis of colon cancer. Mol Ther. 2018;26(5):1287-1298. doi:10.1016/j. ymthe.2018.02.024

15. Cheng B, Rong A, Zhou Q, LncRNA LW. LINC00662 promotes colon cancer tumor growth and metastasis by competitively binding with miR-340-5p to regulate CLDN8/IL22 co-expression and activating ERK signaling pathway. J Exp Clin Cancer Res. 2020;39(1):5. doi:10.1186/s13046-019-1510-7

16. Feng Q, Zhu B, Hu YL, Mao QS, Feng Y. LncRNA HOXA-AS3 promotes gastric cancer progression by regulating miR-29a-3p/LT $\beta R$ and activating NF-אB signaling. Cancer Cell Int. 2021;21(1):118. doi:10.1186/s12935-021-01827-w

17. Ankö ML, Neugebauer KM. RNA-protein interactions in vivo: global gets specific. Trends Biochem Sci. 2012;37(7):255-262. doi:10.1016/ j.tibs.2012.02.005

18. Ma Y, Liu Y, Pu YS, et al. LncRNA IGFL2-AS1 functions as a ceRNA in regulating ARPP19 through competitive binding to miR-802 in gastric cancer. Mol Carcinog. 2020;59(3):311-322. doi: $10.1002 / \mathrm{mc} .23155$

19. Viallard C, Larrivée B. Tumor angiogenesis and vascular normalization: alternative therapeutic targets. Angiogenesis. 2017;20 (4):409-426. doi:10.1007/s10456-017-9562-9

20. Reymond N, D'água BB, Ridley AJ. Crossing the endothelial barrier during metastasis. Nat Rev Cancer. 2013;13(12):858-870. doi: $10.1038 / \mathrm{nrc} 3628$

21. Lamouille S, Xu J, Derynck R. Molecular mechanisms of epithelial-mesenchymal transition. Nat Rev Mol Cell Biol. 2014;15 (3):178-196.

22. Feng Q, Xu D, Zhou M, et al. CDC42EP3 promotes colorectal cancer through regulating cell proliferation, cell apoptosis and cell migration. Cancer Cell Int. 2021;21(1):169. doi:10.1186/s12935021-01845-8

23. Buscail L, Bournet B, Cordelier P. Role of oncogenic KRAS in the diagnosis, prognosis and treatment of pancreatic cancer. Nat Rev Gastroenterol Hepatol. 2020;17(3):153-168. doi:10.1038/s41575019-0245-4

24. Lal N, White BS, Goussous G, et al. KRAS mutation and consensus molecular subtypes 2 and 3 are independently associated with reduced immune infiltration and reactivity in colorectal cancer. Clin Cancer. 2018;24(1):224-233. doi:10.1158/1078-0432.CCR-17-1090

25. Zhang Q, Li H, Mao Y, et al. Apoptotic SKOV3 cells stimulate M0 macrophages to differentiate into M2 macrophages and promote the proliferation and migration of ovarian cancer cells by activating the ERK signaling pathway. Int J Mol Med. 2020;45(1):10-22.

26. Hanahan D, Weinberg RA. Hallmarks of cancer: the next generation. Cell. 2011;144(5):646-674.

27. Antonio N, Bønnelykke-Behrndtz ML, Ward LC, et al. The wound inflammatory response exacerbates growth of pre-neoplastic cells and progression to cancer. EMBO J. 2015;34(17):2219-2236. doi:10.15252/embj.201490147

28. Fridlender ZG, Sun J, Kim S, et al. Polarization of tumor-associated neutrophil phenotype by TGF-beta: "N1" versus "N2" TAN. Cancer Cell. 2009;16(3):183-194. doi:10.1016/j.ccr.2009.06.017

29. Bodogai M, Moritoh K, Lee-Chang C, et al. Immunosuppressive and prometastatic functions of myeloid-derived suppressive cells rely upon education from tumor-associated B cells. Cancer Res. 2015;75 (17):3456-3465. doi:10.1158/0008-5472.CAN-14-3077

30. Farhood B, Najafi M, Mortezaee K. CD8 $(+)$ cytotoxic T lymphocytes in cancer immunotherapy: a review. J Cell Physiol. 2019;234 (6):8509-8521. doi:10.1002/jcp.27782 


\section{Publish your work in this journal}

Cancer Management and Research is an international, peer-reviewed The manuscript management system is completely online and includes open access journal focusing on cancer research and the optimal use of preventative and integrated treatment interventions to achieve improved a very quick and fair peer-review system, which is all easy to use. Visit http://www.dovepress.com/testimonials.php to read real quotes outcomes, enhanced survival and quality of life for the cancer patient. from published authors. 